\title{
EFFICIENT IMAGE RETRIEVAL IN BIG DATABASE USING MULTI FEATURE DESCRIPTOR
}

\author{
Shilpa $\mathbf{R}^{\mathbf{1}}$, Anargha Remesh ${ }^{2}$ \\ ${ }^{I}$ M.Tech Student, Department of CSE, The Oxford College of Engineering, Bangalore, Karnataka, India. \\ ${ }^{2}$ Assistant Professor, Department of CSE, The Oxford College of Engineering, Bangalore, Karnataka, India.
}

\begin{abstract}
Image processing is processing of images by using any form of signal processing using mathematical operations for which an image or a video is input. The image processing output may be an image or a set of parameters based on the image. An image retrieval system for a large database of digital images is a computer system for searching, retrieving and browsing image and obtaining most similar images. From the retrieved image multi features like Color Histogram, HOG Transform, Local Binary feature and Gist. Each of these features need to transfer by applying Heat Kernel function to generate range with each of this feature mentioned to a range form a multiview hash and put it in hash table. Calculate hash for the image and this hash can be map for different images also. The existing method for image retrieval is Hashing technology. Hashing is used to index and retrieve items in a database because it is faster to find the items using the shorter hashed key than to find it using the original value. For most hashing strategies, the performance of retrieval vigorously relies upon the decision of the high-dimensional component descriptor. The disadvantage is Curse of dimensionality and does not search faster. The proposed methods are Multi Feature Descriptor and Multiview alignment Hashing. Multiview alignment hashing approach based on regularized kernel nonnegative matrix factorization(NMF), which can find a compact representation uncovering the hidden semantics and simultaneously respecting the joint probability distribution of data and it can easily searches the similar images in less interval of time and it is effective.. Hash within Hash technology is the future enhancement, the hash size is large partitioning the hash and obtaining the efficient result.
\end{abstract}

Keywords: Multiview Alignment Hashing, Feature Descriptor, NMF, Hash Within Hash.

\section{INTRODUCTION}

Hashing is qualified and most prevalent technique in huge scale database for nearest (closest )neighbor search inserting high- dimensional component descriptors into a similarity preserving hamming space with low measurement. High dimension feature descriptor is most important for performance of retrieval for all hashing methods. When the different type of images are used for hashing, description about single type of feature is not enough. Thus, how to use multiple features of the images for the hashing is a future task. In this paper proposes a novel unsupervised multiview alignment hashing approach based on regularized kernel nonnegative matrix factorization, which can find a compact representation uncovering the hidden semantics and simultaneously respecting the joint probability distribution of data. In previous experiments hashing methods focuses on single feature of the images that is single view hashing. For learning hashing technology they used only one type of characteristic feature descriptor. In existing Images/objects are always represented by using their different kinds of characteristics on different features. Thus it is important for learning hashing methods to include heterogeneous description features. A well known approach for extraction of multi feature descriptors is multiview alignment hashing method. MAH can effectively add multiple characteristics data and extract low dimensional descriptive which is embedded in nonnegative matrix factorization method(NMF). NMF is most famous method for extracting data including grouping, filtering of collaborative object and detection.[1]

\section{RELATED WORK}

Nearest Neighbor Search algorithm.[1] says With in a large database finding the image which is most similar to the query image is Nearest Neighbor (NN) Search. Closeness expressed in terms of a dissimilarity function the less similar the objects, the larger the function values. In every case Approximate NN algorithm doesn't give a guarantee to write the actual closest neighbor, in return for speed improved or savings memory. NMF is most famous method for extracting data including grouping, filtering of collaborative object and detection. The Advantages of NN are Simple to search and easy to understand. The disadvantages are $\mathrm{NN}$ is not scalable due to large sample size in database and NN can not apply for computational complex problems.

Tree Structure algorithms[2] says Tree based search schemas are proposed to partition the data space via various tree structures, among them $\mathrm{KD}$ tree and $\mathrm{R}$ tree are successfully applied to index the data for fast query responses. High- dimensional information can't work with these techniques and does not give faster pursuit contrasted with the direct sweep. Truly saying, dimensionality issues will experience the ill problem in the most of the visionbased undertakings, a large number of measurements on the grounds that visual descriptors have. The Advantages are it 
Supports near(est) neighbor search (similar as before), Works for points and rectangles, Avoids empty spaces, Many variants: X-tree, SS-tree, SR-tree etc, Works well for low dimensions. The Disadvantages are Suffer from curse of dimensionality problems, Less faster compared to linear search.

Locality-Sensitive Hashing algorithm[3] says to retrieve items in a database and to find index items in database faster by using shorter hashed key over original value hashing technique is used. To overcome the disadvantage of Nearest Neighbor query problem which is not scalable due to large data set and suffers from curse of dimensionality uses a hashing technique called Locality-Sensitive Hashing technique(LSH). A similitude pursuit is a gathering of articles/pictures that are described by a significant elements of high dimensional trait space given inquiries as focuses required to discover the closest (most comparable) item to the question. The Advantages are LSH simply employs random linear projects to map data points close to Euclidean space to similar codes, it overcomes the problem of Nearest Neighbor search, to store only one point LSH does not require hash buckets and LSH has better running time guarantee. The Disadvantages are It uses Approximate Near-Neighbor query(ANN), randomly chosen synthetic data can not be used, it is often used to measure the performance of exact similarity search algorithm, found unsuitable for evaluation of approximate algorithm for high data dimensionality, Points $\mathrm{p}, \mathrm{q}$ and distance $\mathrm{d}$ the expected distance outcome is $(\mathrm{O}(\mathrm{d}))$ but standard deviation is only $(\mathrm{O}($ $\sqrt{ } d)$ ). So ANN is not meaningful and there will be no non empty bucket was found so miss ratio will occur.

Single view hashing[4] is the topic on single feature extraction which the previous survey of hashing methods. In single view for learning hashing function only one feature descriptor is used. The objects/images each of has their own characteristics and different features which are represented by several kinds descriptive comprehension. The advantages are Single feature of any image can extract easily. The disadvantages are it is impossible to add the multiple feature descriptors in learning hashing functions. This leads to concentrate on multi features of the images. It can not extract multi features of image like intensity, color information HOG, local binary patterns, color hist and Hash value is more.

The Multi-objective Genetic Programming Projection Pursuit (MOG3P) Algorithm[5] says Highlight extraction is the first and most fundamental step in picture request. Most existing picture game plan strategies use hand-made parts, which are not adaptable for different picture spaces. In this paper, they add to a transformative learning way to deal with thus make range adaptable overall segment descriptors for picture gathering using multiobjective hereditary programming (MOGP). In the configuration, a course of action of primitive 2-D directors are selfassertively combined to fabricate highlight descriptors through the MOGP progressing and a short time later surveyed by two target wellbeing criteria, i.e., the tree multifaceted nature and the order mistake. After the entire progression system finishes, the best-so-far course of action picked by the MOGP is seen as the (near by)perfect segment descriptor obtained. The Advantages are Fascinating low dimensional projections of the dataset where the measure of interestingness. MOGP Enhance the discriminative force and interpretability of the created highlights for information order issues and the objective is to discover an information representation that will build the execution of the learning algorithm(s). The disadvantage is Suffers from curse of dimensionality.

\section{EXISTING SYSTEM}

Locality-sensitive hashing(LSH) is a well known hashing method.[3] It uses nearest neighbor search problem, it will search query which is near to it. LSH maps data points to similar codes based on random linear projections close to a Euclidean space. Single feature is used for similar search for learning hash functions.

\section{PROPOSED SYSTEM}

Single feature[4] can not useful for similar search thus based on Multiview Alignment Hashing uses multi characters of images/objects. MAH efficiently add multiple data and extracts the low dimensional embeds via NMF. Hash is large in size. To decrease the size of hash function into simpler components by using a method called Hash Within Hash. In hash within hash, hash function can be partition into smaller functions so that reduces hash value. System architecture is the conceptual framework design that tells the behavior and structure of a system. An architecture is the applied outline that characterizes the structure and conduct of a framework. An engineering portrayal is a formal depiction of a framework, composed in a way that backings thinking about the basic properties of the framework. It characterizes the architecture parts or building pieces and gives an arrangement from which items can be obtained, and system built up, that will cooperate to execute the general architecture. The System architecture is shown below.

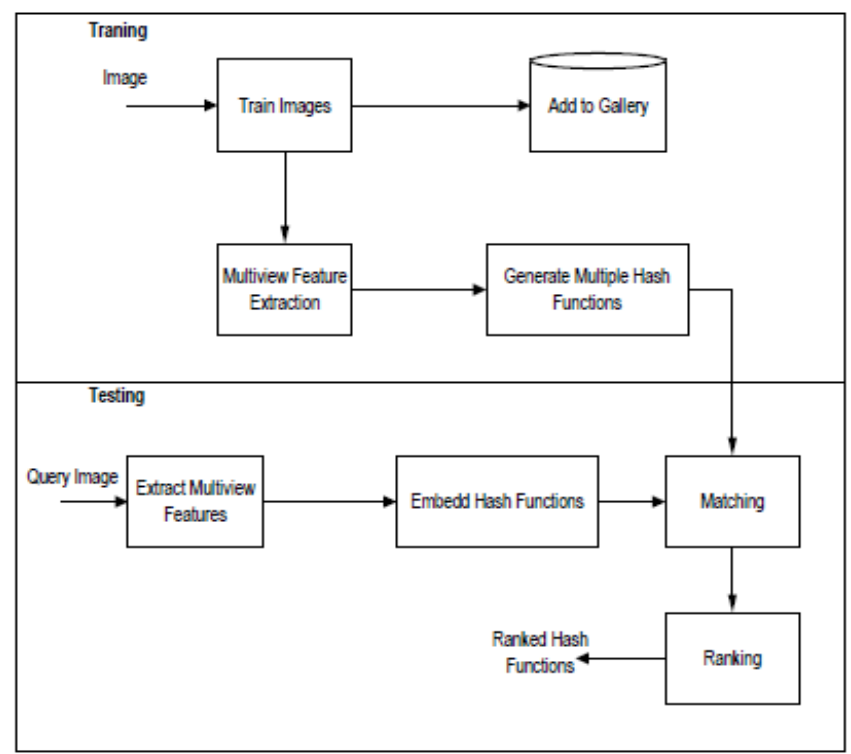

Fig 1: System architecture of Multiview Alignment Hashing. 
MAH: Multi view Alignment Hashing (MAH), which can effectively fuse multiple information sources and exploit the discriminative low dimensional embedding via Nonnegative Matrix Factorization (NMF). NMF is a famous method in data mining which including grouping, filtering collaboration and detection of outlier.[1]

Multi Feature Extraction: the multi features like local binary pattern (LBP), color histogram, histogram of oriented gradients (HOG) are used for image representation. Multi feature extraction is the preprocessing step here. Extracting the multi features is the difficult task.

HOG: in computer vision and image processing a feature descriptor histogram of oriented gradient is used to detect the object. Here HOG is calculated for only grey scale images. Color image is converted in to grey scale image because grey scale have more intensity than color image.

LBP: for classification of image in computer vision local binary pattern feature is used.[5]

Color Histograms: The representation of the distribution of colors in an image, color histogram is used. To represents the number of pixels of images having colors in particular fixed list, a color histogram is used for digital images.

Training Phase: in system architecture during the training phase the images will be trained first which means images will be added to the gallery folder. After adding all the images to the gallery, the multi view feature extraction will be done. All the multiple features will be extracted and calculates the hash value. The generated hash value for all the images will be store in hash table

Testing Phase: during the testing phase, a query image is the input. From that query image extract multi features and calculate the hash value. From the hash value of the query image search the similar images in the hash table. Matching of images are done by MAH. After matching ranking of all images is done by hamming distance sort technology. Finally will get the most similar images for a query image.

\section{RESULTS}

The results of this paper are given below.

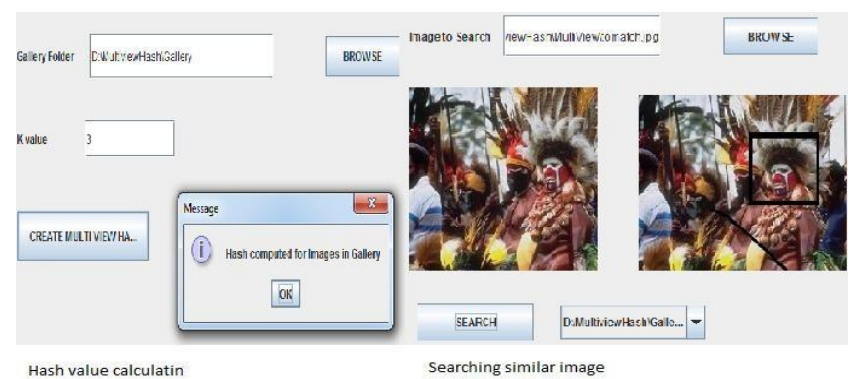

Fig 2: shows retrieving similar images.
Calculating the hash values and retrieval of similar images.

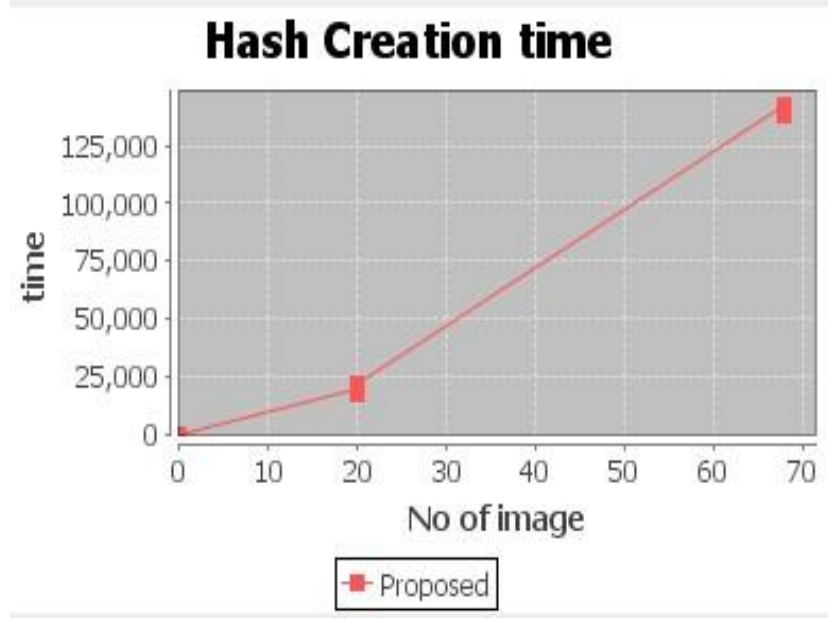

Fig 3: Graph of Hash creation time

The graph showing time(in $\mathrm{m}$ ) verses number of images(20 training example images). Hash creation time is time taken to calculate the hash value for all the images. In above graph showing 20 training images and time taken to calculate hash value for 20 images. The graph says, the time taken for creating hash value for 20 images is 21325 mile seconds.

\section{Search Time}

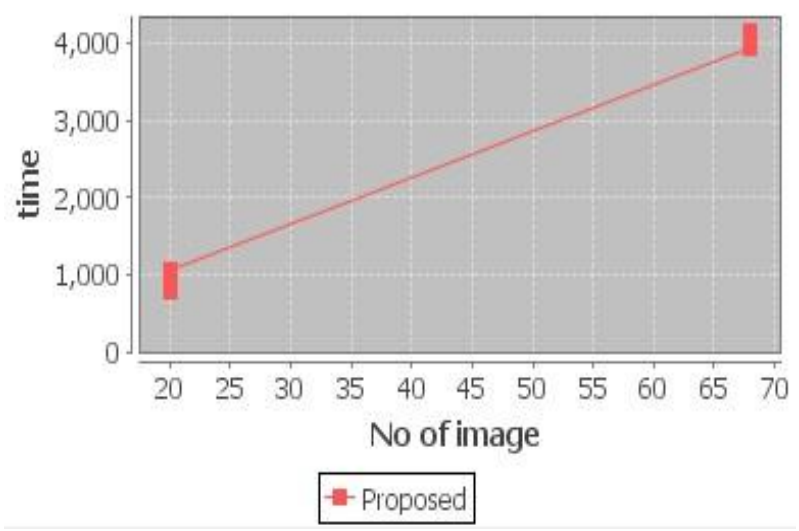

Fig 4: Graph of search time for a given query

Search time is time taken to search the similar images for the given query. In above graph showing 20 training images and time taken to search similar images for a given query. The graph says, time taken for searching similar images for a given query among 20 images is 1100 mile seconds.

\section{CONCLUSION}

Efficient image retrieval in big database using multi feature descriptor is the way to search for the similar images. This paper gives the accurate result and takes less time for hash creation time and searching time. Extracting multiple features of images is more essential for getting most similar images and Hashing is improved by hash within hash technology that is by partitioning of hash values into smaller hash segment. 


\section{ACKNOWLEDGMENT}

It gives me proud privilege to complete this paper under the guidance of Ms. Anargha Remesh by providing all the facilities and helped for smooth progress of this paper. For this I would also like to thank all the Staff Members and Management of Computer Science and Engineering Department, friends and my family members, who have directly or indirectly guided and helped me for the preparation of this Report and gave me an endless support right from the stage the idea was conceived.

\section{REFRENCES}

[1]. Lawrence Cayton and Sanjoy Dasgupta. Nearest Neighbor Search algorithm in "A Learning Framework for Nearest Neighbor Search", jul 1997.

[2]. V. Gaede and O. Günther, Tree Structure algorithms in "Multidimensional access methods", Jun 1998.

[3]. A. Gionis, P. Indyk, and R. Motwani, Locality-Sensitive Hashing algorithm in "Similarity search in high dimensions via hashing",1999.

[4]. J. Han, K. N. Ngan, M. Li, and H.-J. Zhang, Single view hashing in "A memory learning framework for effective image retrieval" , Apr. 2005.

[5]. Liu, and X. Li, The Multi-objective Genetic Programming Projection Pursuit (MOG3P) Algorithm in "Feature learning for image classification via multiobjective genetic programming", Jul 2014. 